\title{
El Desarrollo Urbano Sustentable, ¿Una Utopía o un Proyecto Viable?
}

\author{
Benjamín Alva Fuentes \\ Estudiante de la Maestría en Planeación Urbana-COLMEX
}

\begin{abstract}
Resumen: La degradación del medio ambiente, producto del supuesto "desarrollo" urbano, a ultranza, ha llevado a los especialistas a formular propuestas que disminuyan riesgos y efectos en las sociedades y sobre el territorio. Sin embargo, las "políticas ambientales", como "solución", en el mejor de los casos tratan parcialmente la problemática, y en el peor, quedan muy lejos de la realidad, como simples decisiones poco instrumentadas. El tópico de "moda" que surgió bajo los principios de la "globalización", plantea un intento por instrumentar el "desarrollo sustentable", el cual no podra operar sin antes especificar el contexto territorial y los esfuerzos que implica, reconociendo sus alcances y limitaciones. El "desarrollo urbano sustentable" parece ofrecer una alternativa concreta de los principios de sustentabilidad en el territorio. Los resultados no son de corto plazo, ni mucho menos producto de ejercicios aislados, sólo la suma de esfuerzos sobre el territorio y entre las sociedades, podrá dar una respuesta eficiente y eficaz.
\end{abstract}

Abstract: This paper is focused on analyze environmental degradation as a result of urban "development". The author argues that "environmental policies" as "solution" in the best cases treat the problem partially, and in the worst, remains very far from reality as "wrong" decisions. Also, he indicates that "sustentable development" could not operate, if before it is not specified its territorial context and the efforts that this implicates, recognizing its reaches and limitations. In this context, "sustentable urban development" is seems as an alternative to apply the sustentable principles on territory. However, it is pointed out that outputs are neither short term nor the result of isolated practices, and for this reason the efficient and effective solution to environment problem must emerge from concrete efforts carry out by all countries.

\section{Introducción}

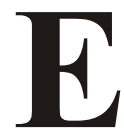

n la actualidad, la problemática ambiental es un tema de análisis recurrente que se vincula a cualquier contexto territorial, en la preocupación por atenuar los efectos negativos producto del acelerado y desarticulado crecimiento urbano, donde destacan la contaminación del aire, agua y suelo, y sus implicaciones resultantes. Las propuestas de solución incorporadas en el discurso ambiental local, pretenden evolucionar paralelamente a los conceptos y categorías de análisis, hechos por los organismos a nivel mundial, tal 
como fue el "ecodesarrollo" en un primer momento, y el "desarrollo sustentable" en la actualidad.

Sin embargo, dadas las particularidades del desarrollo económico, político y social de cada territorio, las propuestas enmarcadas en un contexto global no han sido del todo eficientes al momento de aplicarlas e integrarlas a una realidad específica. El contexto urbano es particularmente un reflejo de las transformaciones del medio ambiente natural, hacía un medio ambiente construido, en un proceso de transformación paulatino a ultranza. Diversas disciplinas pretenden entender y explicar los fenómenos y procesos del medio ambiente; desde la perspectiva de la ecología se describen las formas de comportamiento sobre el territorio visto como un "ecosistema", pero esto no es posible dadas las interrelaciones que existen entre los agentes económicos, políticos y sociales, por lo que no podemos considerar el medio ambiente urbano, socialmente construido como un ecosistema cuya característica básica es la autorregulación, además con ello se aíslan los elementos que interactúan en el tiempo y dan lugar a la conformación de las estructuras institucionales y físicas sobre el territorio, inmersas en un proceso de apropiación diferencial de los recursos. El análisis del medio ambiente, por otro lado, abordado desde las ciencias sociales, nos enfrenta al reto de generar mecanismos operativos eficientes y eficaces que vinculen el "desarrollo sustentable" en un contexto territorial, con diferentes agentes en constante interrelación, garantizando con ello la procuración de un equilibrio medioambiental entre la sociedad-ciudad-naturaleza. La economía en este rubro, desarrolla modelos de optimización y equilibrio, cuyo fin es la disminución de la cantidad de contaminantes que resulte en un incremento del nivel de bienestar para la población, es decir, se ubica básicamente en el campo de la microeconomía, y se concentra en cómo y por qué las personas toman decisiones de consecuencias ambientales, además se ocupa de estudiar y evaluar las políticas económicas y sus alternativas, con el propósito de encontrar un balance entre los impactos ambientales y las metas sociales, con las necesidades del ecosistema en sí mismo (Field, 1995).

De esta suerte, las tres disciplinas principales del estudio del medio ambiente coinciden en el hecho de lograr un equilibrio con diferentes instrumentos, a partir del análisis sistemático de las causas que originan la degradación ambiental. Con base en lo anterior, la idea de estudiar las categorías y conceptos empleados en el discurso 
medioambiental cobra importancia, a efecto de no hacer un empleo excesivo de éstos, e intentar formular políticas coherentes con los planteamientos en los cuales se enmarca, empleando éstas tres principales materias de análisis. Fundamentalmente, la ecología y la economía tienen un nexo más estrecho, en los planos local, regional, nacional y mundial, en un ciclo de causas y efectos.

El "desarrollo sustentable" aparece en la actualidad como una de las alternativas ambientales ampliamente difundidas, el mismo principio de la sustentabilidad emerge en el contexto de la "globalización" como una nueva visión del proceso civilizatorio de la humanidad (Leff, 1996). El concepto de sustenatabilidad surge como el reconocimiento de la función de la naturaleza como soporte, condición y potencial del proceso de producción, de ahí su fuerte vínculo con la racionalidad económica.

Bajo esta lógica, la instrumentación de políticas ambientales es un proceso sumamente complejo, parte desde la nula definición de política y del rubro medioambiental, es decir, se formulan planteamientos cuyo soporte desconocemos si es referido al de una política como tal; el problema de definición de "política ambiental", es latente. Aunado a la imprecisión del contexto territorial donde se debe aplicar, las preguntas entonces son: ¿qué es una política ambiental?, ¿quién o quiénes la deben formular?, ¿cuál es la estrategia a seguir para el cumplimiento de determinados propósitos? y ¿qué instrumentos debe incorporar? En función de lo anterior, intentamos partir desde la acotación del problema, definido como una primera etapa para identificar posibles soluciones, y posteriormente dar paso a la formulación de una "política ambiental", bajo la lógica del proceso de elaboración de "políticas públicas", ya que parece ser el Estado y el gobierno en particular, el ente y agente respectivamente, cuya tarea tiene que ver con la denominada "política ambiental".

El ámbito urbano es el contexto territorial en el cual pueden operar los lineamientos propuestos, ya que es la unidad donde se desarrollan las principales actividades humanas, además el empobrecimiento de los recursos locales condiciona la situación económica de un sinnúmero de regiones, en otras palabras, lo planteado a nivel general sólo tendrá resultados positivos como respuesta a la suma coordinada de lo que opera a nivel local. Los objetivos, lineamientos e instrumentos propuestos, por tanto, deben ser coherentes y en estricto 
apego a las bases planteadas por la corriente del "desarrollo sustentable".

Este documento se constituye por tanto en dos apartados, el primero presenta una breve revisión teórica de los principios y bases que respaldan la posibilidad de definir "desarrollo sustentable", en el medio ambiente urbano, con la respectiva pertinencia y precisión del concepto; y en el segundo apartado se describe un breve diagnóstico general de la problemática ambiental urbana, con el objetivo de ubicar algunos retos y perspectivas, y así enumerar propuestas y alternativas, que pudieran hacer viable la "sustentabilidad urbana".

Cabe mencionar que el presente documento no pretende responder a todos los cuestionamientos que implica el "desarrollo sustentable", ni mucho menos dar respuesta a las preguntas enunciadas respecto de la denominada "política ambiental", sino más bien surge de la preocupación por rescatar los principios que dieron lugar a esta propuesta, los cuales puedan ser un referente en la definición del problema ambiental, y así dar lugar a diagnósticos más acabados, y recuperados posteriormente en objetivos ambientales de tipo urbano, coherentes con los esfuerzos planteados a nivel global. No podemos seguir actuando en forma aislada en las ciudades, sin una planeación integral, debemos ir más allá de tomar "prestado capital del medio ambiente de las futuras generaciones sin intención ni perspectivas de reembolso" (Comisión Mundial del Medio Ambiente y Desarrollo, 1988: 28).

\section{El desarrollo urbano sustentable y su pertinencia en el ámbito urbano}

"Desarrollo sustentable" fue definido por la Comisión Mundial sobre el Medio Ambiente y Desarrollo, creada en 1983, resultado de la preocupación por atenuar los efectos negativos de la tecnología, el desarrollo y la pobreza principalmente, sobre el medio ambiente, advierte que ha llegado el momento de sumar economía y ecología, a fin de que gobierno y sociedad puedan actuar de forma responsable, no sólo respecto a los daños ambientales, sino también en lo que se refiere a las decisiones políticas que los producen, el concepto surge como respuesta a la preocupación de disminuir los efectos propios del "proceso de producción" y su materialización territorial, cuya característica principal es la contaminación y degradación del medio ambiente natural, con implicaciones intra e interregionales, resultado 
de la apropiación excesiva del hombre hacia su ecosistema en forma irracional. El hombre se "desarrolla" y resuelve sus necesidades a ultranza del deterioro de los recursos naturales, esta tendencia a la explotación radica en la enajenación de la sociedad por los principios económicos de concentración y ganancia capitalista, además de intereses políticos, de dominación, y aspectos culturales.

La industrialización y la tecnología han revolucionado el proceso y modo de producción capitalista, en sociedades desarrolladas y subdesarrolladas, permitiendo la generación de nuevos esquemas técnicos de apropiación y administración de los recursos naturales como insumo principal de la sociedad, pero las mismas formas que son causa del adelanto, han provocado tendencias a modificar el planeta y los patrones de vida. Existen incluso restricciones económicas respecto del nivel de desarrollo de un país, que impide se destinen recursos en la procuración de una mejor calidad ambiental, en función de que una economía del tercer mundo tiene problemas como la pobreza, el analfabetismo, el desempleo, etc., que impide se pueda preocupar por cuestiones referentes al medio ambiente. Además "La pobreza es causa y efecto principal de los problemas mundiales del medio ambiente. Es inútil por tanto, tratar de encarar los problemas ambientales sin una perspectiva más amplia que abarque los factores que sustentan la pobreza mundial y la desigualdad internacional" (Comisión Mundial del Medio Ambiente y del Desarrollo, 1988: 23).

De esta suerte, el crecimiento económico visto como proceso, está vinculado estrechamente a las cuestiones del medio ambiente y sus implicaciones. La pobreza condiciona el alto grado de desperdicio de recursos y disminuye la capacidad de afrontar el problema; implica relaciones sociales de producción, patrones de consumo, cultura y en general el modo de vida. "La comisión cree que la pobreza general ha dejado de ser inevitable, la pobreza no sólo es un mal en sí misma. El desarrollo sostenible exige que satisfagan las necesidades básicas de todos y que extienda a todos la oportunidad de colmar sus aspiraciones a una vida mejor. Un mundo donde la pobreza es endémica será siempre propenso a sufrir una catástrofe ecológica o de otro tipo" (Comisión Mundial del Medio Ambiente y del Desarrollo, 1988:29), por tanto la pobreza y la degradación ambiental tienen un vínculo estrecho de carácter explicativo en el proceso de estructuración del territorio como respuesta al nivel de desarrollo. 
De la misma manera, la diferencia de explotación y capacidad de aprovechamiento de los recursos naturales, entre los países industrializados y en vías de desarrollo, es cada vez mayor. La desigualdad, por tanto, es otro problema principal ambiental del planeta, existen diversos factores que producen este empleo diferencial de recursos, como son: el comercio internacional, importante componente de las economías, especialmente en aquellos menos adelantados; en conjunto con un bajo nivel tecnológico, produce problemas de exceso de explotación de recursos del medio ambiente; las "crisis de la deuda" en los países subdesarrollados hacen que la riqueza de sus recursos no se emplee en "vías del desarrollo", sino para satisfacer las obligaciones financieras contraídas con los acreedores extranjeros. Las políticas de atracción de inversión en países subdesarrollados hacen "flexible" el cuerpo normativo, lo cual permite que empresas tanto extranjeras como nacionales altamente contaminantes aprovechen estas condiciones como un factor de localización, en detrimento del medio ambiente, sin considerar que el nivel y proceso tecnológico y de producción de cada empresa, puede incorporar un trato específico a los desperdicios tanto tóxico-industriales como domésticos.

Uno de los planteamientos básicos del desarrollo sostenible, y por tanto de la sustentabilidad ambiental, subrayan la importancia de "definir límites" a la tecnología y a la organización social, impuestos por los recursos naturales en función de la cantidad de desechos de las de las actividades humanas y de absorción de la biosfera, por tanto la tecnología y las actividades humanas pueden ser ordenadas de tal manera que abran el camino a un proceso de crecimiento que incluya elementos tales como: el reciclado de los recursos y la capacidad de absorción de los desechos, haciendo hincapié en las políticas que disminuyan la brecha de la desigualdad y la pobreza. Así el desarrollo sostenible se presenta como un proceso de coherencia entre las necesidades sociales, la explotación de los recursos, el nivel tecnológico, la dirección de las inversiones, la modificación y esfuerzos de las instituciones, en todos y cada uno de los diferentes niveles de gobierno, fundado en un eficiente marco normativo, pero básicamente en la voluntad política.

La inquietud de hacer más operativo el discurso del desarrollo sustentable, establece un proceso de generación de políticas en un contexto cada vez más particular, plantea mecanismos y agentes que 
permitan ser instrumentos de aplicación de objetivos precisos de bienestar social y ambiental. La comisión del medio ambiente y desarrollo, ha centrado su atención en los renglones de población, seguridad alimentaria, desaparición de especies y recursos genéticos, energía, industria y asentamientos humanos, resaltando la interrelación analítica que existe entre todos ellos.

En materia de población, el ritmo de crecimiento total debe ser paralelo o inferior a las tasas de crecimiento de los recursos naturales, aunado a políticas de educación y cultura que modifiquen los patrones de comportamiento tradicionales sobre la administración de los recursos, ya que en la medida que se actúe en el presente, se podrán ver resultados en el futuro, sobre todo en el rubro de los ritmos de crecimiento económico y demográfico.

En el renglón de seguridad alimentaria resalta la importancia del apoyo al sector agrícola, que garantice rendimientos crecientes, desde la etapa de producción hasta el comercio, haciendo hincapié en los pequeños productores, cuyas oportunidades de producción y distribución de sus recursos son inferiores al resto. Además, la orientación para el tipo de producto cultivado, según la capacidad del suelo, puede ser una herramienta técnica ofrecida por el gobierno, "la mayor parte de las naciones en desarrollo precisan sistemas de incentivos más eficaces para estimular la producción, en especial de los vegetales comestibles". (Comisión Mundial del Medio Ambiente y del Desarrollo, 1988: 33). Finalmente, la seguri- dad alimentaria exige atención particular en la etapa de distribución, específicamente en lo relacionado a patrones de consumo y poder adquisitivo de la población.

Entre los especialistas coincide el argumento de que las especies están desapareciendo a ritmos sin precedentes, la diversidad de especies es necesaria para el buen funcionamiento del ecosistema en su conjunto, sin embargo, forman también parte importante de la economía mundial, de ahí que la comisión sugiera la participación del gobierno para frenar la destrucción de las selvas tropicales y otros depósitos de diversidad biológica, balanceado con la reforma en los sistemas de ingreso procedentes de los bosques que disminuyan la deforestación.

El tipo de energía juega un papel importante en la posibilidad de "un desarrollo sostenible", actualmente es cada vez más imprescindible en 
el desarrollo de las actividades humanas modernas, las tasas de utilización en las economías desarrolladas van en aumento, tendencia que difícilmente soportaría el ecosistema de nuestro planeta, sobre todo si se basará en combustibles fósiles no renovables, por tanto, es necesario el empleo de energía segura y duradera, enmarcada en el contexto de la política de eficiencia enérgica nacional, de desarrollo sostenible. "La eficiencia energética sólo permite ganar tiempo mientras se desarrollan medios de bajo consumo de energía basados en fuentes renovables..." (Comisión Mundial del Medio Ambiente y del Desarrollo, 1988: 35).

La industria es uno de los sectores de mayor participación en la economía de las actividades humanas en materia de producción, sin embargo, es también uno de los sectores que generan mayor cantidad de desechos tóxicos al medio ambiente; en este sentido, la microeconomía ambiental analiza las particularidades entre el consumo de insumos, la generación de productos para el desarrollo de las actividades humanas y la producción de desechos, pretende encontrar un nivel óptimo de contaminación bajo el supuesto de que toda actividad industrial genera un nivel determinado de contaminación, y que debido a la dependencia de las actividades industriales no podemos dejar de producir. Bajo esta lógica, la "sustentabilidad de una economía" implica no sólo que siga existiendo, sino más bien usar los recursos renovables de tal modo que el ritmo de extracción sea menor que el ritmo de regeneración natural, y que se mantengan siempre los flujos de residuos al medio ambiente al mismo nivel, o por debajo de su capacidad de asimilación.

Finalmente, los asentamientos humanos ponen de manifiesto en el territorio las interrelaciones de los rubros anteriores, los cuales se hacen más intensos en las áreas urbanas; mismas que por su parte privilegian la concentración económica, social y con ello de explotación de recursos y degradación ambiental, "al terminar el siglo casi la mitad de la humanidad vivirá en centros urbanos" (Comisión Mundial del Medio Ambiente y del Desarrollo, 1988: 38), lo cual intensificará todo este proceso. Específicamente en México, a lo largo del presente siglo la población nacional se ha quintuplicado, aunado a un proceso acelerado de crecimiento en los principales centros urbanos, actualmente las ciudades medias, atraviesan por un proceso de crecimiento demográfico más dinámico, en términos relativos, que el resto de las ciudades. 
Dentro de este contexto, la ciudad ambiental se presenta como una propuesta de viabilidad de los planteamientos del desarrollo sustentable, en términos funcionales y operativos entre ciudades permanentes en el tiempo, que permita mejorar los lugares donde el hombre realiza sus actividades de reproducción social, económica y política, interrelacionadas en el medio ambiente, en cumplimiento de metas que eleven la calidad de vida.

En función de lo anterior, las estrategias de "desarrollo urbano sustentable" se construyen: creando estructuras políticas capaces de promover cambios y beneficios colectivos y evitar la alienación y fragmentación social; asignando funciones en el desarrollo social y medio ambiental; deberes y responsabilidades dentro de las sociedades; y el cambio del papel asignado a los diferentes agentes en el proceso de desarrollo urbano.

Según la propuesta de Satterthwaite (1998), una ciudad sustentable implica diferentes relaciones de articulación y suficiencia, un desarrollo urbano sustentable conlleva la generación y reproducción de recursos para su población, que lejos de establecer un impacto negativo en la región a la que pertenece, promovería el crecimiento y disminuiría las desigualdades en las condiciones de vida, conformaría un ámbito urbano "independiente" en términos de producción de sus insumos, con lo cual dejaría de "importar" recursos, y generar efectos de desequilibrio en las áreas naturales. Mucho menos exportaría en respuesta, desechos del proceso de producción de la ciudad, a otras regiones, por tanto, tienen que buscar una relación congruente $\mathrm{y}$ armónica entre la reproducción de las condiciones generales para el desarrollo, crecimiento social y el aprovechamiento de los recursos en el ámbito natural, pero además generar una cultura de reutilización y trato de desperdicios sólidos, líquidos y gaseosos, resultante del proceso de reproducción urbana. Satterthwaite (1998) presenta la alternativa de considerar cinco líneas específicas que promueven el desarrollo urbano sustentable:

1. Controlar las enfermedades infecciosas y parasitarias, en la medida que se multiplican los vectores y los agentes causantes de enfermedades, el abatimiento de la agenda sanitaria se enfrenta a problemas emergentes de la contaminación ambiental de fuentes fijas y móviles, así como a dos problemas adicionales, los de las enfermedades nuevas y la reaparición de enfermedades 
infecciosas conocidas, que hasta hace poco se consideraban controladas.

2. Reducción de riesgos químicos y físicos en la casa, el lugar de trabajo y la ciudad en general, e incluir políticas de tránsito que minimicen el riesgo de accidentes vehiculares y que protejan a los peatones, acompañadas de estrategias para el empleo del transporte público; los vehículos se convierten en la principal fuente de contaminación atmosférica.

3. Lograr un ambiente urbano de alta calidad, incluye asegurar la cantidad y calidad suficiente de espacios al aire libre, asegurar la provisión de espacios públicos en cada colonia, de forma que respondan a las distintas necesidades y prioridades de los grupos de la población.

4. Minimizar la transferencia de costos ambientales a los habitantes y ecosistemas que rodean a la ciudad, a medida que se disponga de mejores alcantarillados y drenajes en la ciudad, y

5. Consumo sustentable, donde el desempeño ambiental de las personas y empresas que se concentran en la ciudad, deben ser compatibles con las metas a nivel nacional y global, las demandas concentradas de alimento, combustible y materias primas satisfechas en gran medida con importaciones de ecosistemas distantes, de modo que se exige menos a la región circundante, aunado a evitar transferencias de costos hacia el futuro (Satterthwaite; 1988).

La sociedad y su estructura organizacional juegan un papel fundamental en la medida que se constituyen como un problema, objeto e instrumento, de acción de la política ambiental. Bajo esta perspectiva Haughton y Hunter (1994) proponen tres principios para el "desarrollo urbano sustentable":

1. Equidad intergeneracional. Cualquier actividad humana debe considerar la capacidad de futuras generaciones para satisfacer sus necesidades y aspiraciones, referido a un principio futurístico.

2. Justicia social. Esto se refiere a las generaciones actuales donde la pobreza es vista como la primera causa de degradación por el tipo de atención que se da, así como a las condiciones en las que 
se desarrolla. La sustentabilidad requiere un control sobre la distribución de recursos, en atención a las necesidades básicas y aspiraciones comunes.

3. Principio de responsabilidades trasfronteras, se requiere estudiar el ambiente en un contexto global, en la necesidad de controlar la contaminación y la transferencia, los impactos de la actividad humana no deben involucrar un desplazamiento geográfico de los problemas ambientales. Las naciones ricas no deben sobreexplotar los recursos de otras áreas, ya que tiene impactos sobre la economía regional y los ecosistemas. Proporcionalmente, los costos ambientales de actividades urbanas no deben desplazarse a través de límites metropolitanos producto del crecimiento urbano (Haughton y Hunter, 1994).

En términos generales, los problemas de producción, distribución y consumo en el proceso de relaciones sociales, son importantes para el entendimiento de los patrones de contaminación en las ciudades, dentro del contexto para el desarrollo sustentable y sus planteamientos a nivel global.

\section{Diagnóstico general de la problemática ambiental urbana}

Algunas propuestas y alternativas para la viabilidad de la sustentabilidad urbana.

La ciudad se ha transformado en una unidad territorial vital para el desarrollo de las actividades humanas y su reproducción, el sistema económico mundial manifestado en el territorio, es cada vez más un sistema urbano, interrelacionado con sistemas de comunicaciones para la producción y el comercio, las perspectivas de una ciudad dependen entonces del lugar que ocupan en su sistema urbano nacional o internacional, atendiendo a su función económica, por tanto el problema del deterioro ambiental en las ciudades, necesariamente también nos refiere a un proceso de desarrollo económico, las leyes y fuerzas del mercado son más libres en unas sociedades que en otras, el papel del Estado y los márgenes de maniobra varían, la ciudad es una gran consumidora de bienes naturales que evidentemente "importa" y lo hace en exceso e irracionalmente, lo cual se manifiesta a nivel intra e interregional en dos tipos de escasez, la natural y la social, la primera se define por los límites del crecimiento y disposición de recursos, y la segunda es más significativa, por ser socialmente producida se refiere a 
la distribución de los mismos en el sistema socioeconómico. Lo anterior se materializa territorialmente en el proceso de urbanización, que se acentuó a razón del despegue industrial, la urbanización como proceso es condicionado por el modelo económico, en el debate demográfico-ambiental, B. Roberts (1994) lo define como un tema central y secundario: es central porque es un proceso global que no sólo concentra población en ciudades, sino que transforma las áreas rurales con implicaciones en el medio ambiente, y con cambios en los patrones de uso de la tierra, agotando los recursos naturales y contaminando el ambiente físico; y es secundario, ya que no hay razones universales para que la urbanización media entre población y el ambiente. En este proceso, las economías presentan diferente consumo de energía y de recursos, un patrón económico con agentes dispersos consume menos en comparación con un patrón concentrado que consume más, pese a ser de la misma magnitud y con el mismo nivel de tecnología, además, la intensidad en el nivel de urbanización y degradación, estarán sujetas a los lineamientos principales y característicos del modelo económico imperante.

El uso del automóvil como medio de transporte, es causa de una de las principales fuentes de contaminación atmosférica, sin embargo, la falta de una alternativa eficiente de transporte público, y de una estrategia óptima de distribución de viajes según las actividades de la población, no permite que en las ciudades, se reduzca su uso, es decir, los patrones de distribución del empleo y la población en las ciudades a diferencia del campo, produce la necesidad imperante de movilización e intensidad de flujos del lugar de trabajo al hogar, así como a la satisfacción de otras necesidades, el incremento en la energía del transporte de pasajeros refleja las grandes distancias sociales y las tendencias económicas, no se trata solamente de que más personas son propietarias y usan carros, sino que hacen más viajes, y los viajes son cada vez más largos en promedio, por tanto, el problema no sólo radica en los patrones de asignación de rutas de transporte, ni mucho menos en el sentido de las vialidades, sino más bien en los esquemas de distribución de las actividades económicas de la ciudad, que producen la movilidad urbana.

La carencia de un marco jurídico que norme los procesos y constituya la base de un proceso de planeación eficiente, es definitivamente una carencia más en la cual surge el proceso de urbanización e industrialización en los países tercermundistas. A nivel 
interno constituye un factor condicionante para la manifestación y reproducción de problemas ambientales. A nivel externo, la dependencia y atraso tecnológico y científico de los países tercermundistas, respecto de los del primer mundo, conlleva a un desequilibrio entre lo posible y lo deseado (políticas) para abatir la contaminación y en general los problemas del medio ambiente desde el nivel local hasta el contexto global.

Existe una apropiación de los recursos en forma diferencial, resultado de demandas y requerimientos sociales para la reproducción. Los países desarrollados cuentan con una mejor tecnología, que contamina menos, pero los costos de aplicación se elevan sobre la tecnología convencional de los países subdesarrollados, razón por la cual no es rentable ni mucho menos posible incorporarla en el ciclo de producción de estas economías. Los problemas del medio ambiente se explican desde una perspectiva económica y de dependencia de los países según su nivel de desarrollo.

La mayoría de las ciudades del tercer mundo se desenvuelven en un proceso tecnológico y científico limitado, la estructura organizacional de la administración pública es deficiente, y los recursos para la instrumentación de políticas ambientales son escasos, el crecimiento anárquico produce una enorme presión en materia de viviendas y de servicios, e incluso con la idea de atender estos renglones, la calidad de ellos es baja; lo mismo ocurre con la infraestructura necesaria para la reproducción del espacio urbano, el transporte público está deficientemente normado, lo cual permite el servicio de unidades desgastadas que producen altos índices de contaminación, la "oportunidad" de empleo y vivienda que ofrecen las ciudades son esenciales para absorber el crecimiento de población cuyo origen es el campo, en este escenario la concentración urbana es un elemento que intensifica la problemática ambiental. Por tanto, la concentración vista desde la perspectiva ambientalista, no es del todo recomendable, y ésta se intensifica aún más por las políticas gubernamentales macroeconómicas y de precios, lo cual tiene incluso algunas implicaciones económicas, como es: el incremento en la renta del suelo $\mathrm{y}$ aumento en el precio de algunos bienes de consumo con una mayor demanda que el resto.

El papel de las administraciones locales, por su parte, debe ser más innovador en materia macroeconómica, que impulse las actividades 
del campo y disminuya la movilidad rural-urbana, aunado a la generación de políticas coherentes con el sistema urbano nacional, que disminuya la movilidad urbana-urbana, y con ella los efectos de este proceso. Ya que en la mayoría de los países en desarrollo, las estructuras institucionales y jurídicas de los gobiernos locales son inadecuadas, lo cual impide políticas eficientes de desarrollo urbano, económico y social, enmarcadas en un enfoque de planeación tradicional. El problema financiero local se hace cada vez más intenso, por un lado existen efectivamente pocos recursos para dar cumplimiento a las demandas de la población, y por otro lado éstos son deficientemente empleados en el momento de la instrumentación, aunado a la nula o baja coordinación de las autoridades e instituciones locales, con lo cual se disminuye la capacidad económica local para enfrentar problemas del medio ambiente.

En síntesis, los problemas ambientales surgen y se intensifican con el tiempo, por estar inmersos como parte del proceso de urbanización condicionado por el modelo económico; todo en la ciudad está en constante cambio, los lugares de trabajo, recreación y residencia. La industria juega un papel importante como detonante del proceso, el gobierno tiene poca atención en cuestiones de localización de fuentes de empleo y sus impactos, lo cual produce e intensifica la contaminación como parte de la concentración industrial. El crecimiento demográfico, producto de factores de atracción en la ciudad, lleva a la manifestación de altas proporciones de fraccionamientos y centros de negocios que la mayoría de las veces están mal ubicados, o con infraestructura deficiente, que los hace fuentes de desechos sólidos y gaseosos. La interrelación de actividades en el territorio exige constante comunicación y flujo de personas, mercancías y servicios, ante ello, la vialidad y el uso del automóvil mal planificado genera un alto índice de contaminación atmosférica que puede intensificarse por las condiciones geográficas. Las relaciones económicas y sociales involucradas en el proceso de conformación de la ciudad, van más allá de las ya mencionadas, la lista puede aumentar y los problemas también, lo que hay que rescatar principalmente para todo análisis posterior, es que la problemática ambiental es resultado de un proceso de desarrollo de las fuerzas productivas y en general del modelo de producción, manifestado territorialmente y concentrado en la ciudad, el tamaño de ésta, está directamente relacionado con la dimensión y cantidad de problemas ambientales. 
La sustentabilidad urbana, dentro de un contexto operativo, deja de ser una utopía siempre y cuando partamos de un eficiente diagnóstico de la realidad estudiada y formulemos estrategias urbanas fundamentadas en los principios de desarrollo sustentable, los cuales más que considerarlos como algo inconcebible, debemos fijarlo como una imagen-objetivo basada en adecuaciones y políticas paralelas que garanticen su ejercicio.

Los mecanismos eficientes y eficaces de la planeación integral global o local descansan en un cuerpo normativo actualizado y aplicado, en una estructura organizacional de la administración pública sólida, además de la retroalimentación en las diferentes etapas del proceso, la participación institucional y social juega un papel importante para asegurar la coherencia en la administración pública. Cuando se tienen aspectos intertemporales e interregionales que rompen con los planeamientos de mediano y largo plazo, la autoridad local debe garantizar la coherencia en materia de planeación para el desarrollo urbano sustentable, que busque disminuir los grandes intereses particulares que intervienen; la estructura socio-gubernamental debe ser flexible en la medida que logre adaptarse a los problemas actuales, y genere una cultura tanto de la sociedad civil, como de los profesionistas y técnicos que brindan las herramientas a los políticos que toman decisiones, en constante capacitación y conocimiento de los problemas ambientales y los elementos que interactúan en el espacio.

Paralelamente se debe trabajar en el diseño de un sistema de transporte público que sea eficiente en término de las rutas, tiempos y cobertura, que evite problemas de tránsito y tiempos de movilidad, producto de la densidad, usos y distribución del suelo y de las actividades económicas urbanas, que juegan también un papel importante en términos de la eficiencia del transporte, debe existir una coherencia entre grado de densidad demográfica, intensidad de flujos, distancia y motivos de movilidad, así como personal que accede al servicio. Para la propuesta de reestructuración de las ciudades existentes, es necesario considerar las estrategias de desarrollo urbano sustentable, como parte de una estrategia global de desarrollo inmersa en los acuerdos internacionales, realizar programas de sustentabilidad aplicables al territorio urbano. La participación de las comunidades bajo esta propuesta, es de vital importancia, pues en ella recae la aceptación o rechazo y puesta en práctica o eliminación 
respectivamente de una estrategia de desarrollo sustentable a nivel urbano, además constituye una precondición para la continuidad y efectividad del planteamiento original, en diferentes etapas. Las iniciativas se acompañan de elementos educacionales que les fortalecen, entre residentes, autoridades locales e iniciativa privada.

El financiamiento y administración de las políticas de desarrollo urbano sustentable, debe también ser planteado estimulando la inversión pública con prioridades finamente acotadas, y atraer a la ONG's, sociedad civil y la iniciativa privada específicamente.

El principal problema en la aplicación de los principios del desarrollo sustentable, radica en que van en contra de la racionalidad económica y política, e ignoran la confrontación de intereses que se dan en una sociedad de consumo que sustenta a la ciudad industrial, sus principios son más de carácter antropocéntrico, bajo este principio, en la medida que implique mayores costos contaminar, que no hacerlo, el desarrollo sustentable en general, y el abatimiento a la contaminación en particular, será algo posible con resultados alentadores.

\section{Conclusiones y delimitación de la agenda ambiental}

1. La economía y la ecología interactúan de manera destructiva, es entonces a la luz de estas disciplinas principalmente donde se deben formular políticas ambientales urbanas.

2. Se debe considerar como parte del diagnóstico urbano, el origen de los recursos que son consumidos en la ciudad, el destino de los desperdicios, y las políticas actuales para reproducir y mantener los recursos naturales, considerando el ritmo de asimilación del entorno y la capacidad de reproducción.

3. El desarrollo urbano sustentable, deja de ser utopía, en la medida que contextualicemos globalmente los esfuerzos materializados coherentemente en todo el ámbito local en su conjunto.

4. Con un uso excesivo del concepto de desarrollo urbano sustentable, corremos el riesgo de hacerlo poco operativo, debemos tomar en cuenta que los efectos de la problemática ambiental nos están absorbiendo paulatinamente y cada vez se acentúa más la imposibilidad de darles solución, pero avanzamos en planteamientos y alternativas coherentes, el reto 
es sumamente difícil y requiere de tiempo, lo cual es lo único que ya no tenemos.

5. El desarrollo económico definitivamente influye en la posibilidad de respuesta de las alternativas del desarrollo urbano sustentable, y los condiciona a un ciclo, es decir, conforme se puede elevar la calidad de vida en general, nos acercamos a cumplir los propósitos del desarrollo sustentable, pero esto no es posible si paralelamente no ejecutamos acciones que en materia económica nos lleven a un crecimiento y desarrollo. Economía y medio ambiente son dos campos interrelacionados e interdependientes.

6. Las deficiencias ambientales no pueden ni deben ser reducidas a las cuestiones relacionadas con la contaminación local, sino debe implicar desde la exportación e importación de desperdicios tóxicos en un determinado territorio, evaluaciones de impacto ambiental eficientes, control sobre las implicaciones del crecimiento económico a ultranza de la explotación del medio ambiente natural, control sobre el uso del automóvil (bajo la propuesta de alternativas funcionales), y en general analizar los efectos de todos y cada uno de los elementos del medio social construido.

7. Los principios del desarrollo sustentable están definidos, lo que nos corresponde hacer como sociedad y autoridades locales es, hacerlos operativos, según las posibilidades y deficiencias de cada ámbito urbano, algunos requerirán mayor atención en algunos rubros que en otros, pero no podemos soslayar la importancia de ninguno.

8. Finalmente, hay que considerar los impactos ambientales de las ciudades en un contexto explicativo de interrelaciones espacial, social y temporal, es decir, tridimensional de los ecosistemas y momentos que participan en la reproducción de materias primas $\mathrm{y}$ de bienes finales. 


\section{Bibliografía}

Barton, H. (1992), "City transport: estrategies for sustainability", en Sustainable development and urban form, Londres: Pion Limited.

Comisión Mundial del Medio Ambiente y el Desarrollo (1988), Nuestro Futuro Común, Madrid: Alianza Editorial.

Field, B. (1995), Economía Ambiental, Colombia: McGraw-Hill.

Hardoy, J.E. (1992), Environmental problems in third World cities, Londres: Eathscan.

Haughton, G y C. Hunter (1994), Sustainable Cities, Londres: Jessica Kingsley Publishers Ltd.

Leff, Enrique (1996), La insoportable levedad de la globalización: la capitalización de la naturaleza y las estrategias fatales de la sustentabilidad, Programa de Naciones Unidas para el Medio Ambiente, MIMEO.

Nikkamp P. y F. Soeteman (1992), Susteinable development and urban form, Londres: Pion Limited.

Roberts, B. (1994), "Urbanization and the environment", en Rethinking the debate, Westview Press.

Satterthwaite, D. (1998), "Ciudades sustentables o ciudades que contribuyen al desarrollo sustentable", en Revista Estudios Demográficos Urbanos, No. 37, México: El Colegio de México.

White R. y J. Whute (1992), "Cities and the environment: an overview”, en Sustainable cities, Westview Press. 